\title{
Optical Method for Simultaneous High-Resolution Measurement of Heat and Fluid Flow: The Case of Rayleigh-Bénard Convection
}

\author{
Juan F. Torres $\odot,{ }^{1,}{ }^{*}$ Yongling Zhao $\odot,{ }^{2}$ Shuqi Xu๑, ${ }^{1}$ Zhengyu Li, ${ }^{1}$ and Atsuki Komiya ${ }^{3}$ \\ ${ }^{1}$ Research School of Electrical, Energy and Materials Engineering, The Australian National University, Canberra, \\ ACT 2601, Australia \\ ${ }^{2}$ Department of Mechanical and Process Engineering, ETH Zürich, Zürich 8093, Switzerland \\ ${ }^{3}$ Institute of Fluid Science, Tohoku University, Sendai, Miyagi 980-8577, Japan
}

(Received 29 April 2020; revised 23 September 2020; accepted 19 October 2020; published 17 November 2020)

\begin{abstract}
An optical system combining phase-shifting interferometry (PSI) and particle image velocimetry (PIV) is built and verified with simultaneous two-dimensional temperature- and velocity-field measurements of a convective flow. The well-known Rayleigh-Bénard convection in laminar regime in a cubical cavity filled with water is chosen as the experimental validation case. Three-, four-, and six-bucket temporal phase-shifting equations using a rotating polarizer method are tuned under different light-source power conditions, first without PIV, to produce high-resolution phase-shifted data. The results showed that the three-bucket phase-shifting equation is the most robust method over a wide range of laser powers, while the PIV tracers decreased the PSI precision from $1.5 \%$ in the case without tracers to $3.0 \%$ when seeded at $0.02 \mathrm{wt} \%$. The temporal and spatial resolution of the PSI measurement is $0.1 \mathrm{~s}$ and $6.47 \mu \mathrm{m}$, respectively. Owing to the combined PSI and PIV technique, both temperature and velocity characteristics are obtained, unveiling the existence of several flow bifurcations as the Rayleigh number is increased up to $1.06 \times$ $10^{5}$. This optical setup is a potential paradigm shift in heat- and fluid-flow visualization, while having a great potential in biosensor development for concurrent velocity-, concentration-, and temperature-field measurements of aerosols and flows with multicomponent species.
\end{abstract}

DOI: 10.1103/PhysRevApplied.14.054038

\section{INTRODUCTION}

Buoyant flows are ubiquitous in nature $[1,2]$ and engineered systems (e.g., [3-5]). The physics of thermal plumes in buoyant flows has long been one of the central research questions in the fluid mechanics and geophysics communities, owing to its paramount role in convective heat and mass transport phenomena (e.g., [6-9]). A major challenge that limits the exploration of buoyant flows is the lack of experimental diagnostics for simultaneous, high-resolution, and noninvasive temperature- and velocity-field measurements. To underpin experimental explorations, we propose a hybrid optical system in which phase-shifting interferometry (PSI) and particle image velocimetry (PIV) are employed to perform concurrent temperature- and velocity-field measurements.

PSI and PIV are well-established techniques to measure temperature [10] and velocity [11] fields, respectively, but have never been used to determine both flow fields instantaneously. Therefore, high-accuracy measurements are generally limited to either velocity [12] or temperature [13] fields. Another common approach is to complement limited experimental data to validate numerical

\footnotetext{
*felipe.torres@anu.edu.au
}

simulations that are used to have a better understanding of the overall flow behavior $[14,15]$. Although some techniques, which are summarized below, have been developed to measure both temperature and velocity fields, drawbacks in precision might have hindered their widespread adoption. The optical arrangement presented in this study is applied to natural convection in a cubical cavity [16], but it can also be applied to other kinds of thermofluid flows, including internal [17] and external [18] mixed convection flows.

Hiller and Koch [19] conducted experiments on thermal convection in a $38-\mathrm{mm}$ cubical cavity, for a range of Rayleigh and Prandtl numbers $\left(10^{4} \leq \mathrm{Ra} \leq 2 \times 10^{7}\right.$ and $5.8 \leq \operatorname{Pr} \leq 6000$ ), with two opposite vertical walls kept at prescribed temperatures. The velocity and temperature fields are measured using cholesteric liquid crystals (CLCs) suspended as small tracer particles in the medium, which is a technique called digital particle image thermometry/velocity (DPIT/V) [20]. The same heating configuration has been the focus of several theoretical studies [21-23]. Ciofalo et al. [24] used DPIT/V to study Rayleigh-Bénard convection in a shallow rectangular enclosure (aspect ratio 1:2:4) at a moderate Rayleigh number $(\mathrm{Ra} \approx 14500)$. Ozawa et al. [25] employed DPIT/V to measure temperature and flow fields 
of natural convection in a Hele-Shaw cell (HSC). DPIT/V could be easily scalable because the field of view is not limited by the size of a test beam, which is an advantage over conventional interferometry. However, CLCs have a limited range of temperatures that exhibit color reflection [20], generally having an error larger than that of phase-shifting interferometry for similar temperature ranges, e.g., $0.32 \mathrm{~K}$ in DPIT/V [26] versus $0.1 \mathrm{~K}$ in PSI [27]. Besides, DPIT/V does not have the high spatial resolution of recently developed interferometric techniques [13,28-30] (e.g., $3.5 \mu \mathrm{m}$ in Ref. [31]). Pallares et al. [12] conducted a systematic PIV study of Rayleigh-Bénard convection in a $12.5-\mathrm{mm}$ cubical cavity at moderate Rayleigh numbers $\left(\mathrm{Ra} \leq 8 \times 10^{4}\right)$ using silicone oil $(\operatorname{Pr} \approx 130)$. The topology and the velocity fields of all structures are in good agreement with numerical results [22,32]. However, local heat fluxes including heat loss from the sidewalls could not be assessed with PIV. Lee and Kim [33] investigated variations of temperature and velocity fields in a HSC using digital holographic interferometry and a twodimensional PIV system. Their method is a good example of the implementation of both measurement techniques to study convection in enclosures. However, they did not conduct simultaneous measurements, which are useful to better understand the instability properties of flows, such as in the case of transition from laminar to turbulent flow in natural convection [34].

Skarman et al. [35,36] proposed simultaneous PIV and temperature measurements using a CCD-based holographic interferometer, and applied it to Rayleigh-Bénard convection. They used the same camera for the PIV and digital holographic interferometry measurements, and adjusted the focus of a lens to set the measurement region for PIV. Their optical measurement was based on the phase-shift method and algorithms for computational focusing on individual particles. However, in our experience, processing interferograms containing tracers to produce high-quality PIV results is extremely challenging, because (1) the background fringe pattern changes between phase-shifted interferograms, significantly varying the contrast of tracers that is needed for particle identification (see Video S1 within the Supplemental Material [49], where the tracers traveling on black fringes are not visible); (2) there is a larger uncertainty of measurement region corresponding to the focus of the camera lens, in contrast to PIV where the measurement region is determined by the laser-sheet position and thickness. These drawbacks are a motivation for decoupling the images used in the simultaneous velocity and temperature measurements.

With regard to accurate means of measuring temperature fields in a transparent fluid, a PSI technique that uses a rotating polarizer and a three-bucket phase-shifting equation [37] was previously developed, initially to measure Fickian diffusion [38-40] and thermodiffusion [41] phenomena. The optical technique is applied to accurately measure temperature fields in natural convection boundary layers from a vertical hot plate [13], as well as in a cubical cavity filled with a participating and nonparticipating media to evaluate the effects of thermal radiation on natural convection [42]. The same three-bucket phase-shifting equation has been applied to measure thermal boundary layers in forced convection [30], to measure density fields in a transient supersonic heat-transfer flow in a microchannel [43], and to measure methane hydrate decomposition near solid-gas interface [44]. Although the three-bucket phase-shifting equation has been widely used, its precision has never been examined in the context of heat- and mass-transfer measurements.

The significant advantage of digital interferometry (including techniques based on PSI or fast Fourier transform [45]) over other methods lies in its high precision, both in terms of physical quantity measurement (e.g., temperature or concentration) and spatial resolution, which solely depends on the pixel resolution of the camera sensor. For PSI techniques using a micropolarizer array [46] or a prism [31] instead of a rotating polarizer, the temporal resolution depends on the shutter speed of the camera. Although most of the previous works that use PSI also have conjugate heat and fluid flow, PSI cannot provide an accurate measurement of the velocity field. Hence, there is the need to develop an optical technique that provides reliable simultaneous measurements of velocity and temperature fields. Such a technique would deepen our understanding of heat- and mass-transfer phenomena in a variety of scenarios (see Sec. IIIE for an outlook of the proposed technique).

The objective of this study is to develop an optical technique capable of conducting simultaneous measurements of high-resolution two-dimensional temperature and velocity fields. A conventional PIV and a previously developed PSI technique [39] are used to measure velocity and temperature fields, respectively. The method is demonstrated with natural convection in a cubical cavity, but it can be applied to other phenomena such as mixed convection [47] and double-diffusive convection [48], both of which motivated this study.

\section{METHODS}

\section{A. Combined PSI and PIV optical apparatus}

The optical apparatus is shown in Fig. 1. The combined PSI and PIV optical layout is shown in Fig. 1(a), while Fig. 1(b) shows the rotating polarizer angles corresponding to interferograms that are used in different $N$-bucket equations.

\section{Phase-shifting interferometry}

A detailed explanation of the PSI setup, including layout and optical elements, is found in Ref. [39]. 


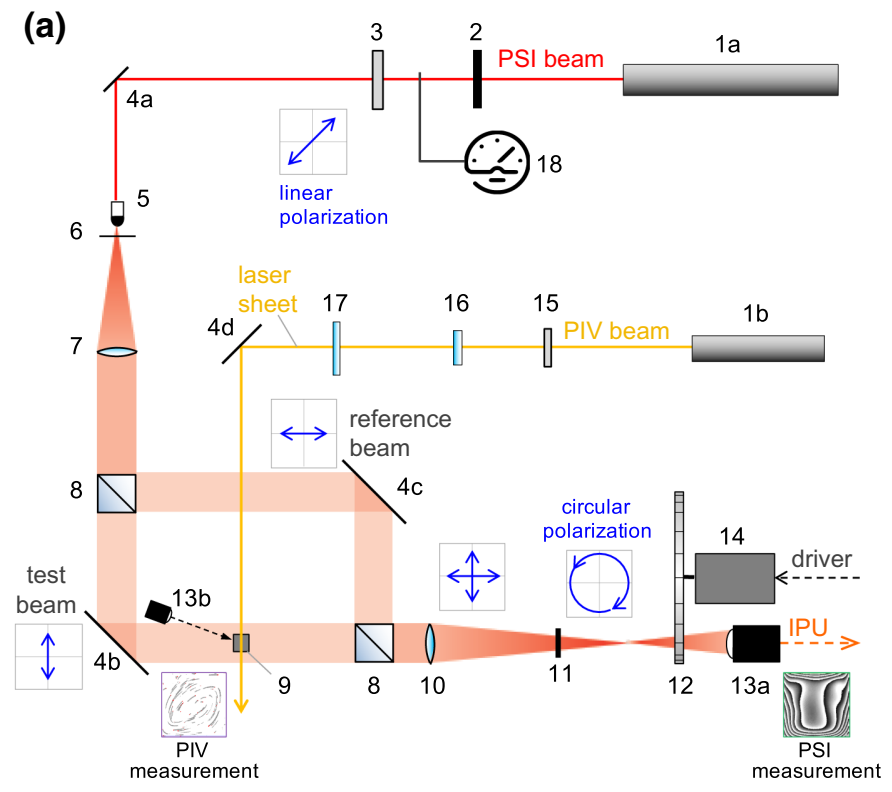

(b) Three-bucket equation image acquisition

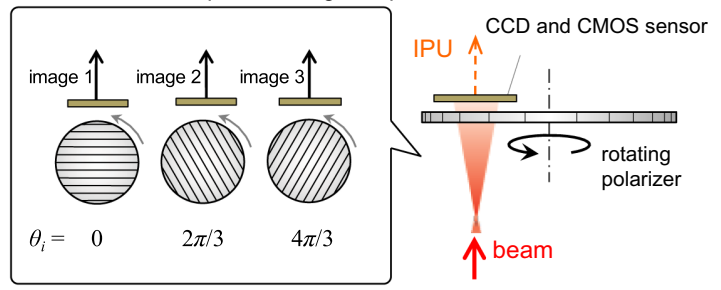

Four-bucket equation image acquisition

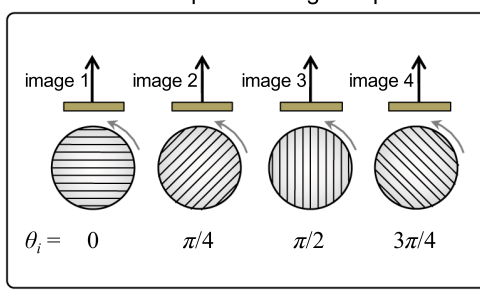

Six-bucket equation image acquisition

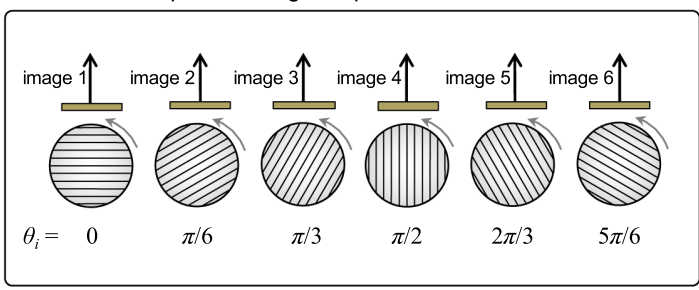

FIG. 1. Schematic of the optical setup. (a) PSI [39] and PIV systems for simultaneous high-resolution measurement of temperature and velocity fields. The PIV system consists of a laser sheet and a camera to register the particle tracers. (1) He-Ne lasers for PSI (1a) and PIV (1b), $\lambda_{\text {beam }}=633 \mathrm{~nm}$ and $10 \mathrm{~mW}$, (2) variable ND filter, (3) linear polarizer, (4) mirror, (5) objective lens, (6) pinhole, (7) spherical lens collimator, (8) polarizing beam splitter, (9) test section: Rayleigh-Bénard cell, (10) focus lens, (11) quarter-wave plate, (12) rotating polarizer, (13) PSI and PIV cameras, (14) stepper motor, (15) ND filter, (16) concave cylindrical lens for beam expansion, (17) convex cylindrical lens for collimating the beam, (18) power meter for tuning the interferometer. Representative measurements for the case-study flow are shown. (b) Phase-shifted data-acquisition method with rotating polarizer for the three-, four-, and six-bucket phase-shifting equations, with polarizer rotation angles of $\theta_{i}$.

A neutral-density (ND) filter is set between the laser and linear polarizer to control the beam intensity going into the optical system (Fig. S1 within the Supplemental Material [49]). The beam intensity is measured as a function of the ND filter angle; note that this is not the intensity incident on the camera because there are optical losses as the beam passes through the system, i.e., reflection, absorption, and spillage. The maximum field of view is ca. $25 \mathrm{~mm}$, but this can be expanded further [42]. The test beam passes through a convective flow that has a refractive-index field, e.g., due to a nonisothermal fluid (Sec. II D). The PSI setup then measures the average temperature along the optical path (OP) within the cell.

After the quarter-wave plate [part 11 in Fig. 1(a)], the orthogonal polarization becomes clockwise and counterclockwise for the test and reference beam, respectively. The phase between reference and test beams in each interferogram, which is recorded with a CCD camera (Hamamatsu, C3077-70) or CMOS camera (Allied Vision, Manta G-235C), can be shifted by using a rotating polarizer. In this study, three $N$-bucket phase-shifting equations [37] - the three-, four-, and six-bucket algorithms listed in Table I- are evaluated in terms of their waviness that represents the precision of the measurement (due to the departure from the planar wavefront), as the laser power is varied. The phase shift $\phi$ at each location $(x, y)$ is evaluated from $N$ number of interferograms using the brightness at the corresponding pixel location $(x, y)$. Any given interferogram $i$ is recorded as the brightness distribution $I_{i}(x, y)$, which is taken at the rotating polarizer angle $\theta_{i}$ indicated in Fig. 1(b) and Table I. Consecutive images are acquired with equivalent time intervals. All components are placed on an optical table with an antivibration control system (photograph shown in Fig. S2 within the Supplemental Material [49]).

\section{Particle image velocimetry}

The PIV system consists of a light source, two cylindrical lenses to expand and collimate the laser beam and form the laser sheet, and a camera (Allied Vision, Manta G-319 with a CMOS sensor resolution of $2064 \times 1536$ pixels, fitted with a Computar MLH-10X lens) to record the motion of tracers in the Rayleigh-Bénard flow. The laser used in 
TABLE I. $N$-bucket family of phase-shifting equations [37] to obtain the phase-shifted data $\phi(x, y)$ from interferograms of intensity $I_{i}(x, y)$ obtained at corresponding rotating polarizer angles of $\theta_{i}$, also indicated in Fig. 1(b). Here, $i=1,2 \ldots, N$ where $N$ is the total number of frames.

\begin{tabular}{lcc}
\hline \hline $\begin{array}{l}\text { No. of } \\
\text { frames }\end{array}$ & Phase-shifting equation & Polarizer angle \\
\hline 3 & $\phi=\arctan \left(\sqrt{3} \frac{I_{2}-I_{3}}{-2 I_{1}+I_{2}+I_{3}}\right)$ & $\theta_{i}=(i-1) \frac{2 \pi}{3}$ \\
4 & $\phi=\arctan \left(\frac{I_{2}-I_{4}}{I_{3}-I_{1}}\right)$ & $\theta_{i}=(i-1) \frac{\pi}{4}$ \\
6 & $\phi=\arctan \left(\sqrt{3} \frac{I_{2}+I_{3}-I_{5}-I_{6}}{2 I_{1}+I_{2}-I_{3}-2 I_{4}+I_{6}}\right)$ & $\theta_{i}=(i-1) \frac{\pi}{6}$ \\
\hline \hline
\end{tabular}

the PIV has the same wavelength as that of the PSI (i.e., $633 \mathrm{~nm}$ ). The PIV measurement region is designated by the location of the laser sheet (ca. $1 \mathrm{~mm}$ thick), in contrast to the PSI temperature measurement that is averaged along the OP. Seeding tracers of ca. $75 \mu \mathrm{m}$ in diameter are introduced in distilled water with two seeding concentrations, $0.02 \mathrm{wt} \%$ and $0.14 \mathrm{wt} \%$, to assess the possible effect of particle density on PSI measurement quality. The material of the seeding particles is styrene acrylate copolymer $\left(1030 \mathrm{~kg} \mathrm{~m}^{-3}\right.$, ELIOKEM, Inc.), which is graded from a bulk supply into various particle sizes. The twodimensional velocity field is obtained with PIVLab [50], an open-source PIV software in MATLAB, using an FFT window deformation algorithm (a discrete Fourier transform correlation with multiple passes and deforming windows). The data is analyzed in three passes, or interrogation windows, with sizes of $200 \times 200$ pixels (pass 1), $140 \times 140$ pixels (pass 2), and $60 \times 60$ pixels (pass 3 ). The smallest pass had a particle density of ca. five particles/window for the low seeding concentration.

In addition to the velocity field, pathlines are obtained in real time with an in-house code written in Processing [51], the same Java library used to obtain the phase-shifted data. The pathlines are produced by filtering the brightness of the image and overlapping the high-brightness pixels of consecutive images for the desired time interval. The pathlines, together with the phase-shifted data, allow a real-time inspection of the flow.

\section{B. Unwrapping and quality assessment of phase-shifted data}

One-dimensional and two-dimensional unwrapping image processing algorithms are developed, first to produce a one-dimensional phase map that can be used to assess the quality of the phase-shifted data, and then to produce two-dimensional phase maps that can be related to the temperature field. A tuning process is first conducted for the one-dimensional phase-shifted data before proceeding with the two-dimensional unwrapping.

\section{One-dimensional unwrapping}

The one-dimensional algorithm follows the procedures described in Refs. [39,41] (Fig. S3 within the Supplemental Material [49]). Wrapped phase-shifted data between 0 and $2 \pi$ is first obtained, but for beams with excessively high or low beam intensities the algorithm does not perform well due to poor-quality interferograms that are saturated or with a large noise-to-signal ratio.

\section{Tuning of phase-shifting interferometer}

The quality of the phase-shifted data is evaluated as a function of the beam intensity, keeping a constant shutter speed. Parasitic losses due to reflection, spillage, and absorption in the system are not quantified. First, the same arbitrary number of horizontal fringes are intentionally set in the interferogram by tilting the test beam mirror. Then, the beam intensity between the variable ND filter and linear polarizer is measured by using a power meter (location shown in Fig. 1) and obtained as a function of the ND filter angle (calibration data shown in Fig. S1 within the Supplemental Material [49]). Next, $N$ number of interferograms $I_{1}, \ldots I_{N}$ are recorded following the process in Fig. 1(b) for a known beam intensity. The interferograms are then processed to obtain the phase-shifted data, whose quality is first evaluated by obtaining the coefficient of determination $R^{2}$ of the unwrapped phase map, as a function of the laser intensity.

\section{Precision assessment}

The precision is further assessed by determining the waviness, which is taken as the deviation of the unwrapped phase map from a linear least-squares fit. Here, a planar wavefront for both reference and test beams is assumed, so that an ideal phase map would be a flat intensity profile. After obtaining the phase map, its quality is evaluated using ten vertical lines at randomly selected horizontal locations to compute the average and standard deviation of $R^{2}$, as well as the waviness with standard deviation. The high-frequency waviness corresponds to the precision of the temperature-field measurement when the relationship of refractive index and temperature is known to a high degree of accuracy. The accuracy of the measurement also depends on the uncertainty of the boundary values for temperature and/or concentration.

\section{Two-dimensional unwrapping}

The two-dimensional algorithm is based on a partitioning technique [52,53] (Fig. S4 within the Supplemental Material [49]). In an isothermal condition, the twodimensional phase maps are related to the nonflat quartz walls of the cell that distort the optical path, producing a curved wavefront. The two-dimensional unwrapping of the phase-shifted data is conducted with an in-house 
program written in Processing [51]. Although several algorithms have been proposed in the literature for an automatic unwrapping of two-dimensional profiles [53-55], in this study we opt for a semiautomatic approach whereby the different layers of a phase-shifted data are manually selected, and then are unwrapped by adding or subtracting $2 \pi$ if the phase gradient at the boundary increases or decreases, respectively. We find that the fully automatic unwrapping algorithm did not perform well when the phase gradient is small in regions of discontinuity $(2 \pi \rightarrow 0)$ due to noise.

\section{Temperature-field measurement}

Two phase maps are needed in order to obtain the twodimensional temperature field (averaged along the optical path), so that only the wavefront deformation due to refractive-index variations is taken into account by the measurement. First, a "background" phase-shifted data is obtained for an isothermal fluid condition. This phase difference accounts for the wavefront deformation due to optical aberrations (imperfections) in the quartz cell. Second, phase-shifted data is obtained in a nonisothermal experiment, which usually has convection. This phase difference represents a wavefront deformed due to both optical aberrations and refractive-index field caused by temperature gradients. After each phase-shifted data is processed with the two-dimensional unwrapping code, the background phase map is subtracted from the convective phase map to obtain a wavefront produced solely by the temperature field (processed explained below in Fig. 4 in relation to the results).

(a)

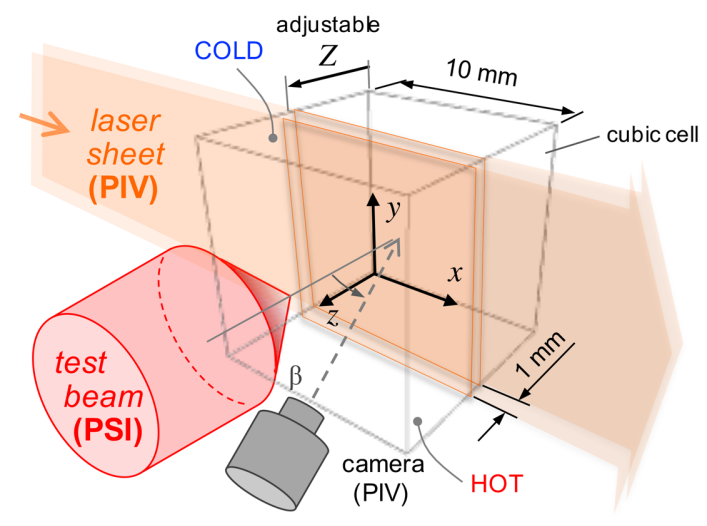

In order to obtain the temperature field, the relationship between refractive index and temperature (or concentration) needs to be known a priori $[30,56]$. The refractive index of water is quasilinear for the temperature ranges and wavelength in this study [57] (i.e., between $22^{\circ} \mathrm{C}$ and $29^{\circ} \mathrm{C}$ for $\lambda=632 \mathrm{~nm}$ ), so a linearly proportional relationship between temperature and phase difference is a good approximation. In contrast, when having large temperature ranges with values below $10^{\circ} \mathrm{C}$, then nonlinearities in the contrast factors need to be included [57,58]. Based on the aforementioned linear approximation in this measurement, the phase map is normalized and the boundary conditions of temperature are applied to the normalized phase map to obtain the two-dimensional temperature field.

\section{Flow-field generator: Rayleigh-Bénard cell}

The Rayleigh-Bénard cell designed for this study is shown in Fig. 2 (photograph shown in Fig. S5 within the Supplemental Material [49]). This cell and its PID temperature-control system follow the same conceptual design as the Soret cell reported by Torres et al. [41], i.e., a parallelepiped cavity confined by quartz sidewalls and upper and lower copper boundaries kept at a constant temperature. The lateral sidewalls allow the passage of light with a low distortion of the wavefront, while the copper allows a fast temperature control due to its high thermal conductivity. The adjustable height of the cavity can be used to study natural convection in parallelepiped cavities with different aspect ratios [59-61].
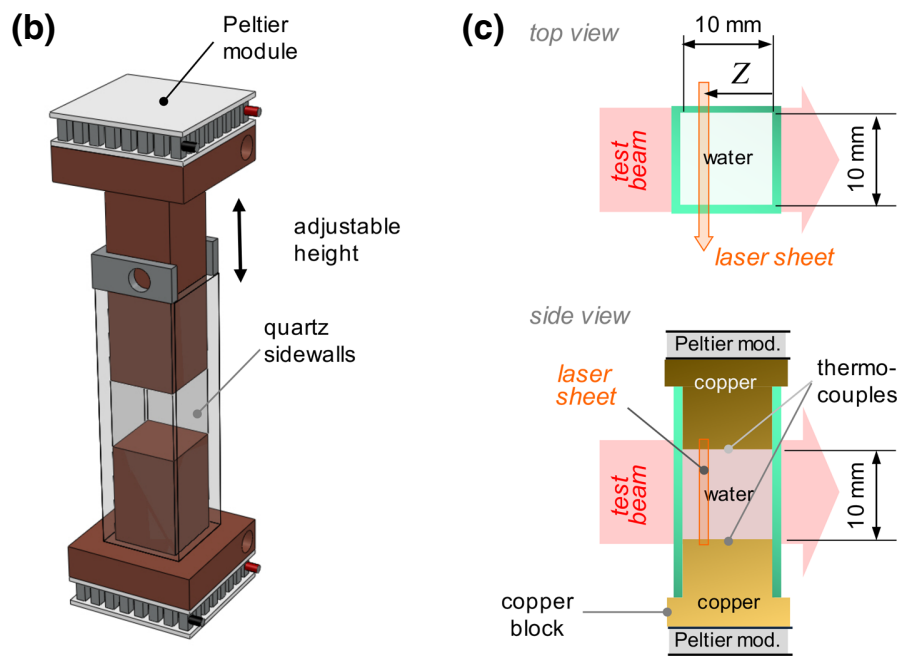

FIG. 2. Rayleigh-Bénard cell, test section used for the proof of concept of the combined PSI and PIV system (Fig. 1). (a) Schematic of the cell, i.e., a cubic volume with a side of $10 \mathrm{~mm}$ filled with water and heated from the bottom wall; a laser sheet is passed through the cell to measure the velocity field at a location $Z$, while the test beam passing along $-z$ is that of the phase-shifting interferometer. (b) CAD model of the cell with upper and lower copper boundaries, and transparent quartz sidewalls. (c) Top and side views of the cell; the quartz sidewalls allow simultaneous measurements of temperature and velocity fields with PSI and PIV, respectively. The temperature at the copper-liquid boundaries is measured by thermocouples and controlled with Peltier modules. A photograph of the cell is shown in Fig. S5 within the Supplemental Material [49]. 
The Rayleigh number Ra - the ratio of buoyancy forces to dissipative factors - in the convection cell is defined as

$$
\mathrm{Ra}=\frac{g \beta H^{3} \Delta T}{\nu \kappa},
$$

where $g$ is the acceleration of gravity, $\beta, \nu$, and $\kappa$ are thermophysical properties of thermal expansion coefficient, kinematic viscosity, and thermal diffusivity; $H$ is the cell height and $\Delta T$ is the temperature difference between the lower and upper boundaries: $\Delta T=T_{\text {hot }}-T_{\text {cold }}$, the lower boundary having a higher temperature than the upper boundary to produce a destabilizing condition. $\mathrm{Ra}$ is controlled by adjusting $T_{\text {hot }}$ and $T_{\text {cold }}$ for a fixed height of $H=10 \mathrm{~mm}$. The temperature difference $\Delta T$ is controlled with a maximum uncertainty of $0.05 \mathrm{~K}$. The room temperature is also set to $T_{\text {mean }}$ to reduce convective heat losses from the sidewalls. A small inclination of ca. $1.5^{\circ}$ about the $-z$ horizontal axis is introduced to favor a clockwise flow solution on the plane visualized by the laser sheet, as in previous theoretical work $[16,23]$.

\section{RESULTS AND DISCUSSION}

\section{A. PSI measurement: quality assessment}

The tuning process of the PSI setup using the three-bucket phase-shifting equation showed that there is a rather large range of laser intensity that yields good-quality phase maps (see Fig. S6 within the Supplemental Material [49]). The reported laser intensity depends on various factors including the properties of the optical elements, magnification, shutter speed of the camera, and attenuation in the working fluid, so its magnitude is only meaningful for the same experimental arrangement. Although outliers of $R^{2}$ sometimes occurred, these are probably due to a beam misalignment caused by a double reflection in the ND filter. Other factors that might have affected the quality of the phase-shifted data include beam alignment, linear polarization angles (initial beam should be set to $\pi / 4$ as indicated in Fig. 1), principal axis of the quarter-wave plate (should be set to $\pi / 4$ to change linear to circular polarization), parasitic reflections that introduce noise or amply the intensity, nonperpendicular incidence of beam on the rotating polarizer (depicted in Fig. S7 within the Supplemental Material [49]) and soiling condition on the optical surfaces. Following the interferometer tuning process, the precision of the three-, four-, and six-bucket phase-shifting equations (Table I) is evaluated based on the $R^{2}$, its standard deviation, and waviness of the phase map. The evaluation is first conducted without the test cell (shown in Fig. 2), and then in the presence of an isothermal test cell with and without tracers.

The phase-shifted data acquired using the three-, four-, and six-bucket algorithms are shown in Fig. 3(a) in the case (a)

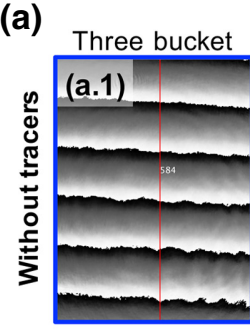

unwrapped
phase map
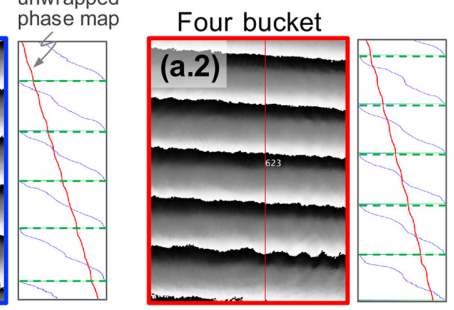

\section{(b)}

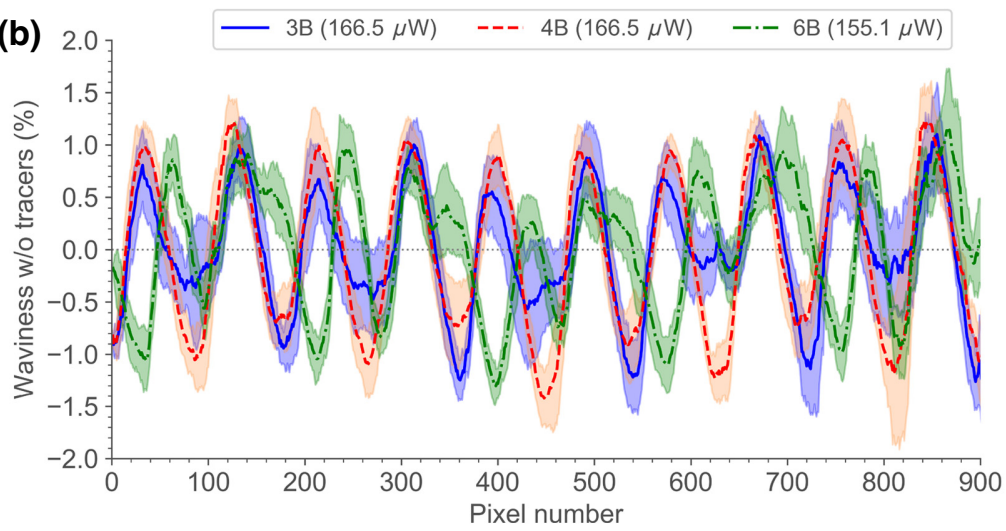

(c)
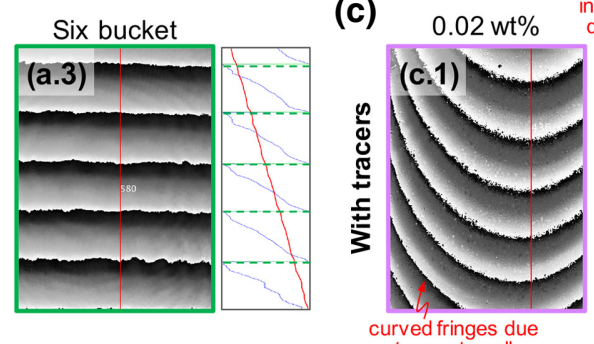

increased noise
due to tracers

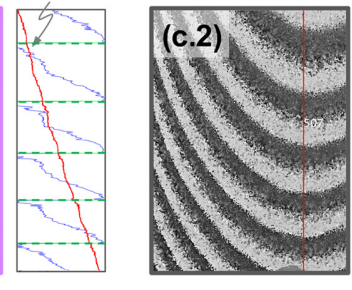

(d)
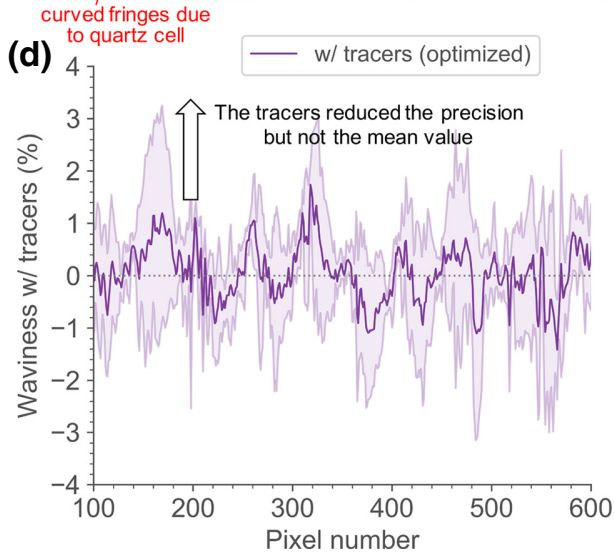

FIG. 3. Evaluation of the phase-shifted data. (a) Data with relatively good quality obtained from the three $N$-bucket phase-shifting equations after tuning; the laser power for each equation is indicated in the legend. The insets indicate the wrapped phase shift (blue), unwrapped phase map (red), and discontinuity location (dashed green) along a selected vertical line on the phase-shifted data. (b) Waviness corresponding to each $N$-bucket equation without test cell; shaded region shows the standard deviation. (c) Phase-shifted data of solution with tracers containing two concentrations. (d) Corresponding waviness of the dilute seeded water (isothermal). 
without the presence of the test cell. The precision assessment shown in Fig. 3(b) indicates that the waviness of the measurements using any phase-shifting equation yields a fluctuation of within $\pm 1.5 \%$ of the total phase-map difference. The data showed that the three-bucket algorithm produced measurement quality of $R^{2}>0.9996$ and $\sigma<$ $1.5 \times 10^{-4}$ for the laser power range between 155.1 and 204.7 $\mu \mathrm{W}$ (Fig. S6 and Table S1 within the Supplemental Material [49]). Although some measurements failed possibly due to multiple reflections, the data produced by the four-bucket algorithm is generally of good quality as well, but with a larger standard deviation. The six-bucket algorithm also produces high-quality measurements for the low and high laser-power ranges, but not so good-quality data for the midpower range. This suggests that using more images helps compensate the loss of quality when using interferograms with a rather high noise-to-signal ratio, but at the same time using more images increases the chance of introducing errors by optical misalignment (e.g., see Figs. S7 and S8 within the Supplemental Material [49]). In addition to its robust high precision for a wide range of laser intensity, the three-bucket phase-shifting equation eases the computational effort, allowing a real-time image processing with a better time resolution. Therefore, the three-bucket phase-shifting equation is chosen in this study to measure temperature fields.

The phase-shifted data using the three-bucket phaseshifting equation for a test cell filled with isothermal water containing tracers is shown in Fig. 3(c). Two concentrations are reported under the same laser-power value: 0.02 $\mathrm{wt} \%$ (c.1) and $0.14 \mathrm{wt} \%$ (c.2). The concentration of 0.02 $\mathrm{wt} \%$ (c.1) is found to be suitable for both PIV and PSI, which had a spatially averaged waviness close to the case without tracers $( \pm 1 \%)$ and a standard deviation of about $\pm 3 \%$ (ca. twice that of the case without tracers), as shown in Fig. 3(d). In contrast, the water seeded at a high tracer concentration severely affected the PSI measurement [the noise is obvious despite Fig. 3(d) not being laser tuned]. Therefore, we demonstrate that PSI can be used concurrently with PIV, without a significant compromise of precision if the seeding particle concentration is properly chosen. Although the PSI measurement quality is not evaluated quantitatively as a function of tracer concentration per OP unit length, the low seeding particle concentration is sufficient for the PIV measurements reported here.

\section{B. Simultaneous phase-shifted data and pathlines: transient flow}

In an experiment to visualize the transition from transient to steady convection, the upper copper wall is kept at a lower constant temperature of $25^{\circ} \mathrm{C}$ (the same as the initial temperature of the fluid), while the lower wall is heated to $29^{\circ} \mathrm{C}$ within ca. $80 \mathrm{~s}$ but with a small overshoot [Fig. S9(c) within the Supplemental Material [49] ]. Images for
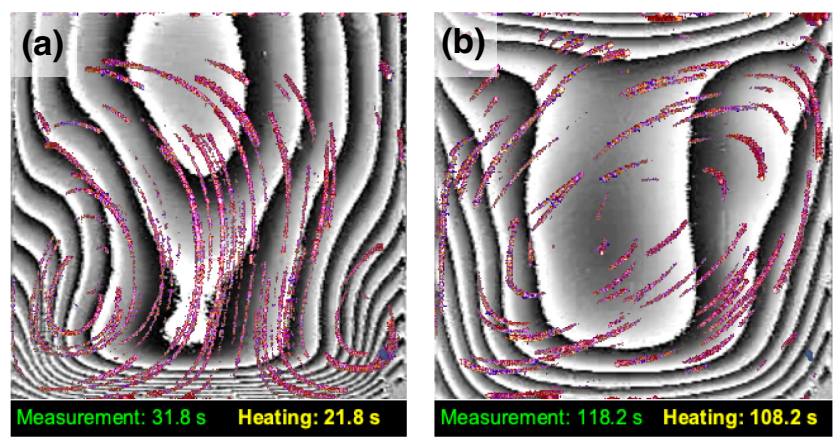

VIDEO 1. Pathlines at $Z=5 \mathrm{~mm}$ overlapped with the phaseshifted data in an experiment of transient Rayleigh-Bénard convection. The cold (top) wall is kept at $T_{\text {cold }}=25^{\circ} \mathrm{C}$ while the hot (bottom) wall is set to $T_{\text {hot }}=29^{\circ} \mathrm{C}$; the initial temperature of water is $T_{\text {cold }}$. Clip (a): transient condition showing how the heat is transported by the fluid motion, which is caused by a destabilizing temperature gradient near the lower boundary. Clip (b): quasisteady state for fluid and heat flow in a clockwise direction within the cell. Snapshots and time series of PID control are found in Fig. S9 within the Supplemental Material.

both disperse tracers and interferograms are simultaneously acquired and further postprocessed. Video 1 shows the interesting relationship between fluid and heat flow, by overlapping both pathlines ( $Z=5 \mathrm{~mm} ; 3$-s trajectory) and phase-shifted data. Large temperature gradients near the bottom wall destabilized the fluid, which subsequently carried heat within the cavity (clip a), reaching steady state for both fluid and heat flow (clip b). Progressive phaseshifted data and pathlines in the transient and steady-state cases are further analyzed (see Fig. S9(a) and (b) within the Supplemental Material [49]).

At $t=0 \mathrm{~s}$ (heating time), there is no flow due to an isothermal condition, but vertical fringes are observed in the phase-shifted data, indicating that the quartz sidewalls are not perfectly flat. As the temperature of the bottom wall increased, horizontal fringes close to the heated boundary gradually appeared; despite the destabilizing temperature gradient, flow motion had barely commenced due to viscous effects, as evidenced by a weak flow motion that exhibited short pathlines. At $17.7 \mathrm{~s}$, the fluid flow is evident with the appearance of two rolls in the lower part of the cavity, where the destabilizing temperature gradients are largest. The flow field seemed to have entered a quasisteady state after ca. $95 \mathrm{~s}$, forming the single clockwise roll. The phase-shifted data in steady state (Video 1, clip b) indicates where the temperature gradients are largest, i.e., near the upper and lower walls; likewise, the lack of new fringes appearing in the center of the phase-shifted data indicates a temperature field that is locally homogeneous.

\section{Concurrent temperature and velocity fields: steady flow}

The process of acquiring the temperature field (averaged along the beam path) is shown in Fig. 4 for the 
(a) isothermal

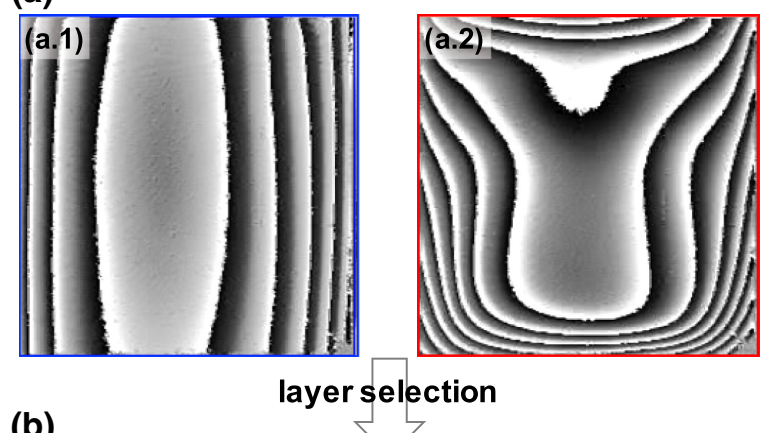

(b)

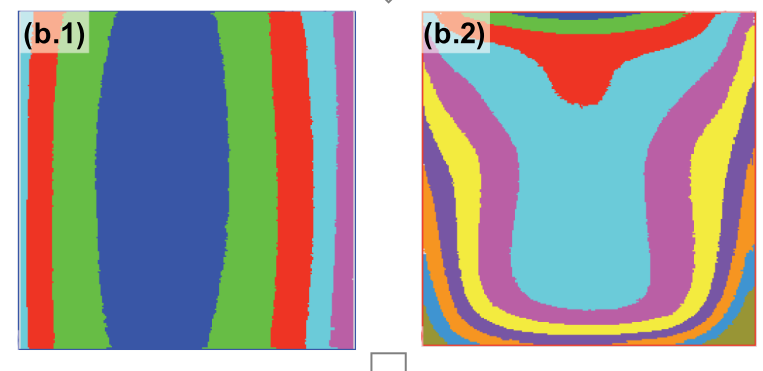

(c)

phase map due to optical aberrations

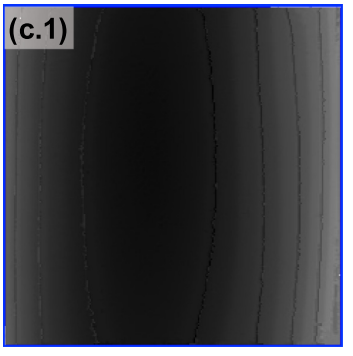

(d) wavefront due to temperature field

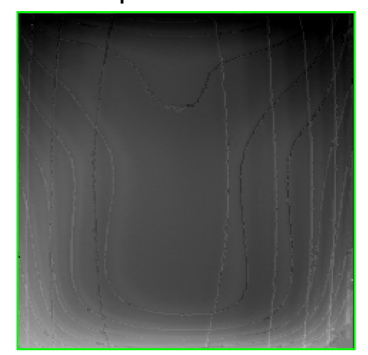

phase map with convective flow

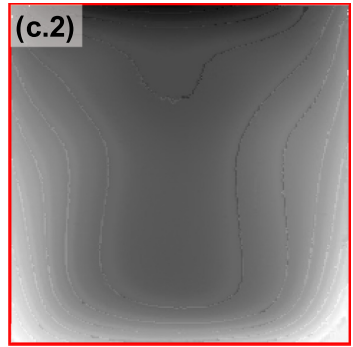

(e)
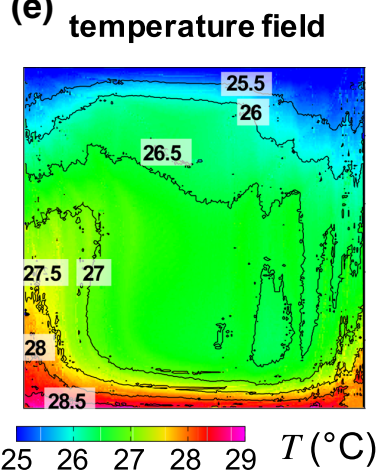

FIG. 4. Processing of phase-shifted data to obtain the temperature field averaged along $z$. (a) Wrapped phase-shifted data for an isothermal $\left(25^{\circ} \mathrm{C}\right.$; background image) and nonisothermal condition; vertical fringes appeared in the former due to optical aberrations caused by nonflat sidewalls. (b) Layers identified by the two-dimensional semiautomatic unwrapping algorithm. (c) Unwrapped phase-shifted data (or phase map) for both the isothermal background and natural convection cases. (d) Phase map due to temperature gradients only, obtained after subtracting the background phase map. (e) Unfiltered two-dimensional temperature field. quasisteady Rayleigh-Bénard flow of Video 1. The semiautomatic unwrapping algorithm successfully detected the layers in the phase-shifted data for both isothermal and nonisothermal conditions, as shown in Figs. 4(a) and 4(b). These data are unwrapped and subsequently subtracted to obtain the corresponding temperature field, as shown in Figs. 4(c)-4(e). The precision of this measurement is $0.17 \mathrm{~K}$, which corresponded to $3.0 \%$ of the maximum temperature difference of $4 \mathrm{~K}$ in the Rayleigh-Bénard cell with tracers (Sec. III A) and the uncertainty related to the PID temperature control (Sec. II D). A marginal noise can be seen in the low gradient region where the $28.5^{\circ} \mathrm{C}$ isotherm in Fig. 4(e) passes through, which is a noise consistent with the measurement precision. Note that in order to compare simulation $[22,23]$ and experimental results, the temperature field in the numerical simulation needs to be integrated along the OP.

Concurrent measurements of temperature and velocity fields are shown in Fig. 5(a) for steady Rayleigh-Bénard convection, as the Rayleigh number $\mathrm{Ra}$ is increased from 0 to $1.06 \times 10^{5}(\Delta T$ of $5.5 \mathrm{~K})$. Preliminary experiments showed that, at the measured mean temperatures, the flow became steady $15 \mathrm{~min}$ after increasing $\Delta T$ by $0.5 \mathrm{~K}$. Therefore, the data shown in Fig. 5 is taken after this time period. The mean temperature is kept at $T_{\text {mean }}=25^{\circ} \mathrm{C}$ while the temperature difference $\Delta T$ between the lower and upper walls is gradually increased from 0 to $5.5 \mathrm{~K}$ by increments of $0.5 \mathrm{~K}$, except for an additional data point for $\Delta T=0.25 \mathrm{~K}$. The temperature fields are spatially smoothed by applying a Gaussian filter. Although the spatial filtering helps remove the noise of the measurement (i.e., at the center of the cell), it is not useful for determining large heat fluxes at the boundary due to a decrease in spatial resolution, which is needed for large temperature gradients. The velocity field captured at $Z=7.5 \mathrm{~mm}$ is also plotted in Fig. 5(a). A plane different to that in Video $1(Z=5 \mathrm{~mm})$ is chosen since it is convenient for depicting the expected symmetry breaking [23].

Based on the PSI and PIV results in Fig. 5(a), at least four characteristic flows in the laminar steady-state regime are identified. The first characteristic flow [Fig. 5(a.1)] corresponds to the near-no-flow condition obtained for $\Delta T \leq 0.5 \mathrm{~K}$, where viscous forces mostly overcome buoyant forces within the fluid, damping its motion to a large extent. The small inclination of the cavity of ca. $1.5^{\circ}$ (about the $-z$ axis) is predicted to replace the no-flow solution found in Rayleigh-Bénard convection below the first instability threshold with a leading branch having a weak flow below the same threshold [23]. The isotherms plotted every $0.05 \mathrm{~K}$ for $\Delta T=0.5 \mathrm{~K}$ suggest a weak plume rising from the left side of the cavity, which is consistent with the slight inclination. The second characteristic flow (a.2) corresponded to a single vortex whose eye is located at $(x, y) \approx$ $(7,5) \mathrm{mm}$, which contradicts theoretical predictions that 
(a)
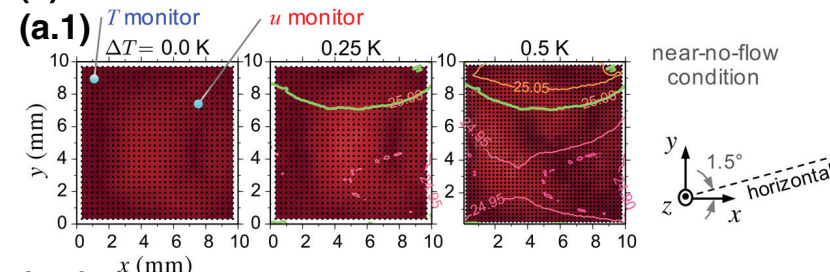

(a.2)
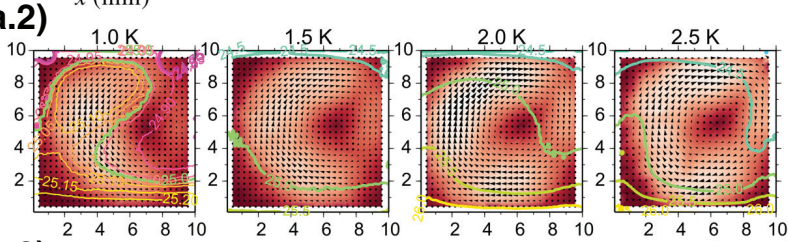

(a.3)
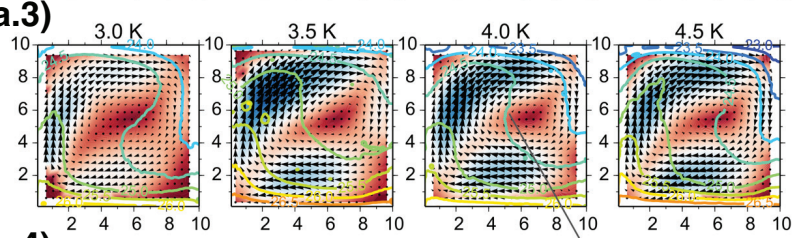

(a.4)

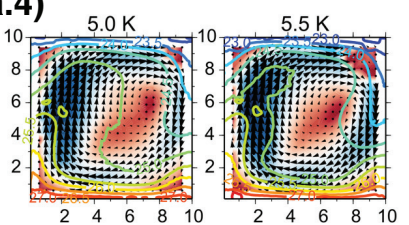

(b)

Temperature difference $(\mathrm{K})$

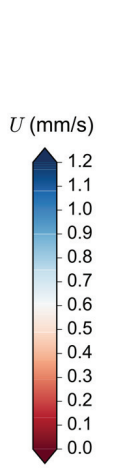

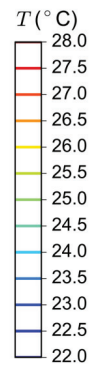

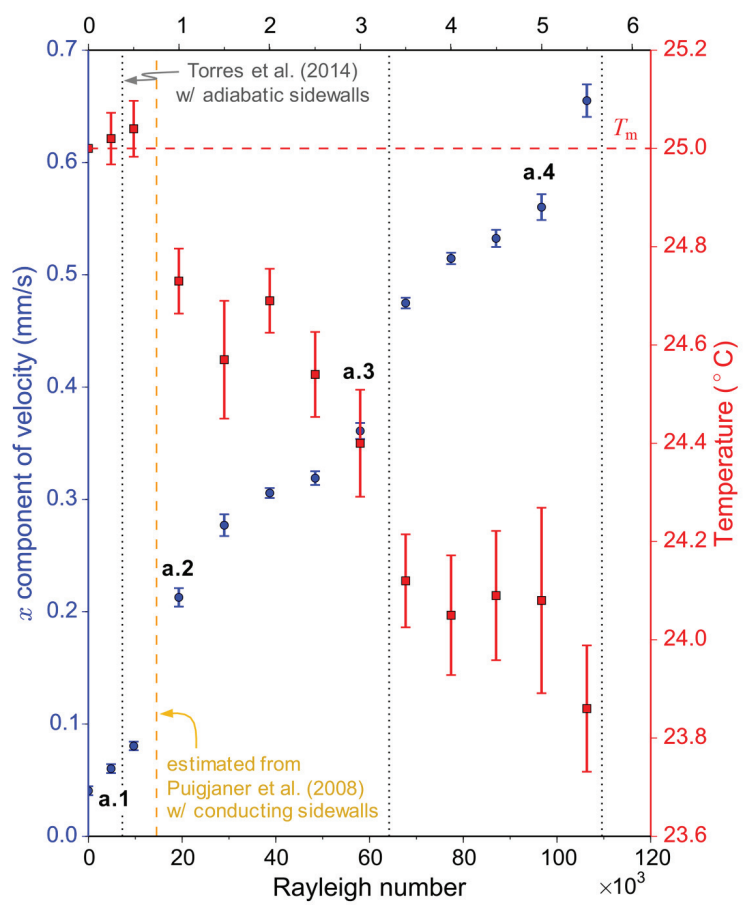

FIG. 5. Characterization of Rayleigh-Bénard convection in a cubical cavity using the proposed optical technique. See Table S2 within the Supplemental Material, for the Rayleigh number Ra values. The vertical dotted lines indicate the critical Rayleigh number $\mathrm{Ra}_{c}$ reported in Ref. [23] for an inclination of $1.5^{\circ}$, adiabatic sidewalls, and $\operatorname{Pr}=5.9$, while the vertical dashed line (orange) shows $\mathrm{Ra}_{c}$ estimated from Ref. [22] for an inclination of $1.5^{\circ}$ and conducting sidewalls. (a) Temperature field on the $x-y$ plane averaged along the $z$ direction; overlapped with the $x-y$ velocity profile on the plane $Z=7.5 \mathrm{~mm}$. (b) Empirical bifurcation diagram using the $x$ component of the velocity at $(x, y)=(7.5,7.5) \mathrm{mm}$ (left axis) and the temperature value at $(x, y)=(1,9) \mathrm{mm}$ (right axis) as a function of Ra, which is linearly dependent on the temperature difference $\Delta T$. The error bars for velocity correspond to the standard deviation of ten consecutive PIV measurements (with a time interval of $0.2 \mathrm{~s}$ ), whilst the error bars for temperature correspond to a $3.0 \%$ of $\Delta T$ experimental error accounting for PSI precision plus the standard deviation in the boundary temperature during the PID control.

place a single roll solution with a horizontal linear axis passing through the cavity center [22,23]. Inaccurate numerical assumptions for the perfectly insulating or conducting boundary conditions, as well as marginal imperfections in the internal geometry of the cavity, might have contributed to such a discrepancy. The third characteristic flow (a.3) corresponded to a clockwise rotating flow with a low-velocity region stretching along the diagonal $y=x$. A rather steep increment of velocity is observed at $(x, y) \approx(3,7) \mathrm{mm}$, which twisted the isotherms about the axis of rotation, producing positive vertical temperature (stabilizing) gradients for $\Delta T \geq$ $4 \mathrm{~K}$ near the center of the cavity. The fourth characteristic flow (a.4) showed a significant increase of velocity at $x \approx 2 \mathrm{~mm}$ and $y=[2,8] \mathrm{mm}$. The observed positive temperature gradients are thought to have stabilized the flow along the central vertical plane $(x=5 \mathrm{~mm})$, contributing to the increment of momentum near the left boundary.

\section{Empirical bifurcation diagram: flow transitions as $\mathrm{Ra}$ is increased}

The results presented in Fig. 5(a) are summarized by plotting the amplitude of the flow as a function of Ra. The amplitude is taken as the $x$ component of the velocity (u) and temperature $(T)$ at arbitrary locations, as indicated in Fig. 5(a.1). Figure 5(b) shows the empirical bifurcation diagram that unveils four branches and three bifurcations as $\mathrm{Ra}$ is increased until 106400 . The values of $T$ changed drastically as a critical Rayleigh number $\mathrm{Ra}_{c}$ is encountered. The dotted vertical lines in Fig. 5(b) correspond to the theoretical results for $\mathrm{Ra}_{c}$ in Ref. [23] for a cavity inclination of $1.5^{\circ}$ from the horizontal plane (one edge is kept horizontal) with adiabatic sidewalls and a Prandtl number of $\operatorname{Pr}=5.9$, which corresponds to that of distilled water at $27^{\circ} \mathrm{C}$ (a slightly larger value than the experiments reported in Fig. 5). Note that neither the perfectly adiabatic or conducting sidewall boundary condition found in the literature (e.g., Refs. [22,23]) is experimentally achieved, because 
the sidewalls are made of quartz, whose thermal conductivity of $k=1.4 \mathrm{~W} \mathrm{~m}^{-1} \mathrm{~K}^{-1}$ is about 3 times that of water $\left(0.58 \mathrm{~W} \mathrm{~m}^{-1} \mathrm{~K}^{-1}\right)$.

The experimental results in Fig. 5(b) show that the first instability threshold is encountered in the range $9.7 \times$ $10^{3}<\mathrm{Ra}<19.3 \times 10^{3}$. Torres et al. [23] predicted a value of $\left.\mathrm{Ra}_{c}\right|_{\theta=1.5^{\circ}}=7.25 \times 10^{3}$ for adiabatic sidewalls, which is approximately $114 \%$ larger than the critical value for the horizontal configuration $\left.\mathrm{Ra}_{c}\right|_{\theta=0^{\circ}}=3388.5$. Based on theoretical predictions for a cubical cavity with perfectly conducting sidewalls [22,62-64], the first instability threshold occurs at $\left.\operatorname{Ra}_{c}\right|_{\theta=0^{\circ}} \approx 6799$, so we can expect that the critical value in the slightly tilted configuration becomes $\left.\operatorname{Ra}_{c}\right|_{\theta=1.5^{\circ}} \approx 14.5 \times 10^{3}$ (i.e., ca. $114 \%$ larger than the horizontal case), which falls within the observed range in Fig. 5(b). Theoretically, only the first $\mathrm{Ra}_{c}$ in Rayleigh-Bénard convection is Prandtl number independent, so a one-on-one comparison with theoretical predictions in the literature is not currently possible for secondary bifurcations in tilted cavities with conducting sidewalls.

\section{E. Outlook of the proposed technique}

PSI has been applied to study various physical phenomena. In convective heat transfer, PSI has proven to be useful to investigate natural [13], mixed [30], and forced [43] convection. In mass transfer, PSI is useful to investigate Fickian diffusion of electrolytes [39] and protein aqueous solutions [40], Soret effect for organic species [65], including proteins [41], hydrate decomposition [44,66], and multicomponent mass diffusion $[38,56]$. Information on the velocity field, additional to the temperature or concentration field, will contribute to further expand our knowledge on conjugate heat- and mass-transport processes.

The proposed PSI and PIV technique is demonstrated for concurrent measurement of temperature and velocity fields, but it could also be applied to measure combined concentration and velocity fields. This has useful applications in drug-delivery systems [67] in which the drug is first transported through arteries and veins, where convection is dominant [68], and then reaches the organs through capillaries, where mass diffusion dominates [69]. PSI has already been shown to have exceptional spatial and temporal resolution of up to 3.5 and $3.3 \mu \mathrm{s}$ [31,44], when using an Arbaa prism instead of a rotating polarizer. Temporal resolution could also be improved by using a micropolarizer array-based phase-shifting interferometer [46,70]. Therefore, the proposed technique can be applied to quantify microfluidic devices, such as a thermophoretic separation device [71] or a microfluidic biosensor for detection of disease, including severe acute respiratory syndrome coronavirus 2 [72].

With regard to the limitations of the proposed technique, since it is governed by the same principles of optical interferometer and PIV, then it shares the same intrinsic drawbacks. For example, it cannot be applied to opaque fluids. Furthermore, phase-shifted data may not be unwrapped throughout the whole field of view in regions where the gradients of fluid temperature or concentration are too large, e.g., in a thermal plume where the temperature difference with the ambient fluid is substantial (see Video S1 within the Supplemental Material [49]). Large temperature gradients entail two technical problems: (a) a fringe density that cannot be resolved with the finite pixel resolution of the camera, and (b) excessive beam deflection. Mitigation strategies to resolve the high-density fringe region - without necessarily reducing the large temperature gradients - include shortening the optical path within the cell (Torres et al. [39] increased the optical path to visualize low differences in refractive index $n$ ) or changing the working fluid to have a more favorable contrast factor $(\partial n / \partial T$ or $\partial n / \partial C$; where $C$ is the concentration). The later approach should not significantly change the Prandtl number and/or Schmidt number of the fluid for temperature and/or concentration measurements, respectively. Furthermore, we show that the PSI measurement is affected when the PIV particle density is excessive [Fig. 3(c)]. In such a case, the seeding concentration needs to be reduced, but this could affect the accuracy and/or spatial resolution of the PIV measurement. For a very low particle density, particle tracking velocimetry could be used as a substitute for PIV.

\section{CONCLUSIONS}

Despite convection entailing the concurrent transport of both momentum and energy, most of the experimental visualization techniques developed so far focus on either heat and mass or fluid flow, represented by temperature and concentration and velocity fields, respectively. This study demonstrates an optical technique that combines a precise phase-shifting interferometry and a conventional particle image velocimetry system to produce simultaneous measurements of temperature and velocity fields.

Simultaneous measurements of temperature and velocity fields reveal the relationship between concurrent transport of momentum and thermal energy in laminar Rayleigh-Bénard convection inside a cubical cavity, which serve to validate the applicability of the proposed optical technique. In addition to its simplicity due to the fewer number of images, the three-bucket phase-shifting equation is more robust than the four- and six-bucket phase-shifting equations when using a rotating polarizer. A tuning process of the interferometer yields a highprecision measurement of ca. $3.0 \%$ of the maximum temperature difference in the flow, with a high spatial resolution of ca. $6.47 \mu \mathrm{m}$; the presence of particles reduces the PSI precision from ca. $1.5 \%$, corresponding to the case without tracers. A simultaneous real-time measurement of the flow using pathlines and phase-shifted data 
is conducted; both measurements are overlapped to visualize the concurrent heat and fluid flow in a transient regime (Video 1). Caution is necessary when interpreting the results, since the temperature field is an average along $z$ (horizontal coordinate along the optical path) whilst the velocity field is a measurement at a given location. Buoyancy within the Rayleigh-Bénard cell is strengthened by gradually increasing the Rayleigh number from the isothermal configuration until $106400(\Delta T: 0 \rightarrow 5.5 \mathrm{~K})$, a range in which three bifurcations and four branches are identified. A good agreement is obtained with the available theoretical predictions near the first instability threshold.

This instrument is a powerful tool to investigate conjugate heat- and mass-transfer phenomena. It could play a useful role in the development of new separation devices and biosensors, as well as in the understanding of fundamental fluid mechanics such as flow instability in natural convection boundary layers. The developed instrument could be further improved focusing on strong threedimensional flows (e.g., by applying tomography techniques) and turbulent conditions.

\section{ACKNOWLEDGMENTS}

Part of the work has been carried out under the Collaborative Research Project of the Institute of Fluid Science, Tohoku University. Funding from the 2019 ANU Global Research Partnership Scheme for the project Exchange on multi-scale convective heat transfer is acknowledged. The kind support of Professor Shigenao Maruyama and Dr Daniel Henry during Dr Torres' PhD is acknowledged.

[1] G. O. Hughes and R. W. Griffiths, Horizontal convection, Annu. Rev. Fluid Mech. 40, 185 (2008).

[2] L. Cheng, J. Abraham, Z. Hausfather, and K. E. Trenberth, How fast are the oceans warming? Science 363, 128 (2019).

[3] T. Tsuji, S. Saita, and S. Kawano, Dynamic Pattern Formation of Microparticles in a Uniform Flow by an On-Chip Thermophoretic Separation Device, Phys. Rev. Appl. 9, 24035 (2018).

[4] M. Caccia, M. Tabandeh-Khorshid, G. Itskos, A. R. Strayer, A. S. Caldwell, S. Pidaparti, S. Singnisai, A. D. Rohskopf, A. M. Schroeder, D. Jarrahbashi, T. Kang, S. Sahoo, N. R. Kadasala, A. Marquez-Rossy, M. H. Anderson, E. Lara-Curzio, D. Ranjan, A. Henry, and K. H. Sandhage, Ceramic-metal composites for heat exchangers in concentrated solar power plants, Nature 562, 406 (2018).

[5] C. Rolin, B. Song, and S. R. Forrest, Mass Transport Through the Carrier Gas Boundary Layer in Organic Vapor Phase Deposition, Phys. Rev. Appl. 1, 1 (2014).

[6] K. L. Chong, Y. Yang, S. D. Huang, J. Q. Zhong, R. J. Stevens, R. Verzicco, D. Lohse, and K. Q. Xia, Confined Rayleigh-Bénard, Rotating Rayleigh-Bénard, and
Double Diffusive Convection: A Unifying View on Turbulent Transport Enhancement through Coherent Structure Manipulation, Phys. Rev. Lett. 119, 1 (2017).

[7] V. Mathai, X. Zhu, C. Sun, and D. Lohse, Flutter to tumble transition of buoyant spheres triggered by rotational inertia changes, Nat. Commun. 9, 1792 (2018).

[8] Y. C. Xie, G. Y. Ding, and K. Q. Xia, Flow Topology Transition via Global Bifurcation in Thermally Driven Turbulence, Phys. Rev. Lett. 120, 214501 (2018).

[9] Z. Wang, V. Mathai, and C. Sun, Self-sustained biphasic catalytic particle turbulence, Nat. Commun. 10, 1 (2019).

[10] C. Shakher and A. K. Nirala, A review on refractive index and temperature profile measurements using laserbased interferometric techniques, Opt. Lasers Eng. 31, 455 (1999).

[11] F. Scarano and M. L. Riethmuller, Advances in iterative multigrid PIV image processing, Exp. Fluids 29, S051 (2000).

[12] J. Pallares, F. X. Grau, F. Giralt, M. P. Arroyo, F. X. Grau, and F. Giralt, Experimental laminar Rayleigh-Benard convection in a cubical cavity at moderate Rayleigh and Prandtl numbers, Exp. Fluids 31, 208 (2001).

[13] E. Shoji, A. Komiya, J. Okajima, and S. Maruyama, Development of quasi common path phase-shifting interferometer for measurement of natural convection fields, Int. J. Heat Mass Transfer 55, 7460 (2012).

[14] J. F. Torres, F. Ghanadi, Y. Wang, M. Arjomandi, and J. Pye, Mixed convection and radiation from an isothermal bladed structure, Int. J. Heat Mass Transfer 147, 118906 (2020).

[15] Y. Zhao, C. Lei, and J. C. Patterson, PIV measurements of the K-type transition in natural convection boundary layers, Exp. Therm. Fluid Sci. 101, 62 (2019).

[16] J. F. Torres, D. Henry, A. Komiya, and S. Maruyama, Transition from multiplicity to singularity of steady natural convection in a tilted cubical enclosure, Phys. Rev. E - Stat., Nonlinear, Soft Matter Phys. 92, 023031 (2015).

[17] K. Wu, B. D. Welfert, and J. M. Lopez, Complex dynamics in a stratified lid-driven square cavity flow, J. Fluid Mech. 855, 43 (2018).

[18] F. Ghanadi, J. F. Torres, M. Arjomandi, and J. Pye, Flow structure and convective heat transfer in a bladed structure under wind conditions, Int. J. Heat Fluid Flow 85, 108676 (2020).

[19] W. J. Hiller and S. Koch, Three-dimensional structures in laminar natural convection in a cubic enclosure, Exp. Therm. Fluid Sci. 2, 34 (1989).

[20] D. Dabiri, Digital particle image thermometry/velocimetry: A review, Exp. Fluids 46, 191 (2009).

[21] S. Wakashima and T. S. Saitoh, Benchmark solutions for natural convection in a cubic cavity using the high-order time-space method, Int. J. Heat Mass Transfer 47, 853 (2004).

[22] D. Puigjaner, J. Herrero, C. Simó, and F. Giralt, Bifurcation analysis of steady Rayleigh-Bénard convection in a cubical cavity with conducting sidewalls, J. Fluid Mech. 598, 393 (2008).

[23] J. F. Torres, D. Henry, A. Komiya, and S. Maruyama, Bifurcation analysis of steady natural convection in a tilted 
cubical cavity with adiabatic sidewalls, J. Fluid Mech. 756, 650 (2014).

[24] M. Ciofalo, M. Signorino, and M. Simiano, Tomographie particle-image velocimetry and thermography in RayleighBénard convection using suspended thermochromic liquid crystals and digital image processing, Exp. Fluids 34, 156 (2003).

[25] M. Ozawa, U. Müller, I. Kimura, and T. Takamori, Flow and temperature measurement of natural convection in a Hele-Shaw cell using a thermo-sensitive liquid-crystal tracer, Exp. Fluids 12, 213 (1992).

[26] M. Behle, K. Schulz, W. Leiner, and M. Fiebig, Colorbased image processing to measure local temperature distributions by wide-band liquid crystal thermography, Flow, Turbulence Combust. 56, 113 (1996).

[27] V. Stoica and P. Stephan, Phase shift interferometry for accurate temperature measurement around a vapor bubble, Exp. Heat Transfer 20, 261 (2007).

[28] A. Komiya and S. Maruyama, Precise and short-time measurement method of mass diffusion coefficients, Exp. Therm. Fluid Sci. 30, 535 (2006).

[29] A. Mialdun and V. Shevtsova, Development of optical digital interferometry technique for measurement of thermodiffusion coefficients, Int. J. Heat Mass Transfer 51, 3164 (2008).

[30] E. Shoji, R. Nakaoku, A. Komiya, J. Okajima, and S. Maruyama, Quantitative visualization of boundary layers by developing quasi-common-path phase-shifting interferometer, Exp. Therm. Fluid Sci. 60, 231 (2015).

[31] E. Shoji, A. Komiya, J. Okajima, H. Kawamura, and S. Maruyama, High-speed phase-shifting interferometry using triangular prism for time-resolved temperature measurement, Appl. Opt. 54, 6297 (2015).

[32] J. Pallares, I. Cuesta, and F. X. Grau, Laminar and turbulent Rayleigh-Benard convection in a perfectly conducting cubical cavity, Int. J. Heat Fluid Flow 23, 346 (2002).

[33] S.-J. Lee and S. Kim, Application of holographic interferometry and 2D PIV for HSC convective flow diagnostics, Meas. Sci. Technol. 15, 664 (2004).

[34] Y. Zhao, P. Zhao, Y. Liu, Y. Xu, and J. F. Torres, On the selection of perturbations for thermal boundary layer control, Phys. Fluids 31, 104102 (2019).

[35] B. Skarman and J. Becker, Simultaneous 3D-PIV and temperature measurements using a new CCD-based holographic interferometer, Flow Meas. Instrum. 7, 1 (1996).

[36] B. Skarman, K. Wozniak, and J. Becker, Digital in-line holography for the analysis of Bénard-convection, Flow Meas. Instrum. 10, 91 (1999).

[37] D. Malacara, Optical Shop Testing (John Wiley \& Sons, Hoboken, 2007), Vol. 59.

[38] A. Komiya, J. Torres, and S. Maruyama, Measurement of mass diffusion coefficient of multi-component system in aqueous media by phase shifting interferometer, Defect Diffus. Forum 297-301, 624 (2010).

[39] J. F. Torres, A. Komiya, E. Shoji, J. Okajima, and S. Maruyama, Development of phase-shifting interferometry for measurement of isothermal diffusion coefficients in binary solutions, Opt. Lasers Eng. 50, 1287 (2012).

[40] J. F. Torres, A. Komiya, J. Okajima, and M. Shigenao, Measurement of the molecular mass dependence of the mass diffusion coefficient in protein aqueous solutions, Defect Diffus. Forum 326-328, 452 (2012).

[41] J. F. Torres, A. Komiya, D. Henry, and S. Maruyama, Measurement of Soret and Fickian diffusion coefficients by orthogonal phase-shifting interferometry and its application to protein aqueous solutions, J. Chem. Phys. 139, 074203 (2013).

[42] T. Kogawa, E. Shoji, J. Okajima, A. Komiya, and S. Maruyama, Experimental evaluation of thermal radiation effects on natural convection with a Rayleigh number of 108-109 by using an interferometer, Int. J. Heat Mass Transfer 132, 1239 (2019).

[43] Y. Takahashi, L. Chen, J. Okajima, Y. Iga, A. Komiya, and $\mathrm{S}$. Maruyama, Interferometric measurement and numerical comparisons of supersonic heat transfer flows in microchannel, Appl. Therm. Eng. 109, 582 (2016).

[44] Y. Kanda, H. Komatsu, J. Okajima, S. Maruyama, and A. Komiya, Evaluation of rate-determining step of methane hydrate decomposition by measurement of transient heat and mass transfer near solid-gas interface, Int. J. Heat Mass Transfer 149, 119191 (2020).

[45] A. Mialdun and V. Shevtsova, Measurement of the Soret and diffusion coefficients for benchmark binary mixtures by means of digital interferometry, J. Chem. Phys. 134, 044524 (2011).

[46] M. Novak, J. Millerd, N. Brock, M. North-Morris, J. Hayes, and J. Wyant, Analysis of a micropolarizer array-based simultaneous phase-shifting interferometer, Appl. Opt. 44, 6861 (2005).

[47] J. F. Torres, F. Ghanadi, I. Nock, M. Arjomandi, and J. Pye, Mixed convection around a tilted cuboid with an isothermal sidewall at moderate Reynolds numbers, Int. J. Heat Mass Transfer 119, 418 (2018).

[48] S. Maruyama, K. Takahashi, A. Komiya, and M. Behnia, Measurement of transient double diffusive convection and crystal growth using real-time phase-shifting interferometer, JSME Int. J. Ser. B 44, 561 (2001).

[49] See Supplemental Material at http://link.aps.org/supplemen tal/10.1103/PhysRevApplied.14.054038 for more details on experimental setup and method.

[50] W. Thielicke and E. J. Stamhuis, PIVlab - towards userfriendly, affordable and accurate digital particle image velocimetry in MATLAB, J. Open Res. Software 2, e30 (2014).

[51] D. Shiffman, Learning Processing: A Beginner's Guide to Programming Images, Animation, and Interaction (Morgan Kaufmann, Burlington, 2009).

[52] J. J. Gierloff, Phase unwrapping by regions, Curr. Dev. Opt. Eng. II 0818, 2 (1987).

[53] S. Liu, Regional phase unwrapping method based on fringe estimation and phase map segmentation, Opt. Eng. 46, 051012 (2007).

[54] M. A. Herráez, D. R. Burton, M. J. Lalor, and M. A. Gdeisat, Fast two-dimensional phase-unwrapping algorithm based on sorting by reliability following a noncontinuous path, Appl. Opt. 41, 7437 (2002).

[55] M. A. Herráez, J. G. Boticario, M. J. Lalor, and D. R. Burton, Agglomerative clustering-based approach for two-dimensional phase unwrapping, Appl. Opt. 44, 1129 (2005). 
[56] A. Mialdun, I. Ryzhkov, O. Khlybov, T. Lyubimova, and V. Shevtsova, Measurement of Soret coefficients in a ternary mixture of toluene-methanol-cyclohexane in convection-free environment, J. Chem. Phys. 148, 044506 (2018).

[57] A. H. Harvey, J. S. Gallagher, and J. M. H. L. Sengers, Revised formulation for the refractive index of water and steam as a function of wavelength, temperature and density, J. Phys. Chem. Ref. Data 27, 761 (1998).

[58] A. N. Bashkatov and E. A. Genina, in Saratov Fall Meeting 2002: Optical Technologies in Biophysics and Medicine $I V$ (International Society for Optics and Photonics, Saratov, 2003), Vol. 5068, p. 393.

[59] S. J. M. Linthorst, W. M. M. Schinkel, and C. J. Hoogendoorn, Flow structure with natural convection in inclined air-filled enclosures, J. Heat Transfer 103, 535 (1981).

[60] J. Colombani, H. Dez, J. Bert, and J. Dupuy-Philon, Hydrodynamic instabilities and Soret effect in an aqueous electrolyte, Phys. Rev. E 58, 3202 (1998).

[61] J. Colombani and J. Bert, Holographic interferometry for the study of liquids, J. Mol. Liq. 134, 8 (2007).

[62] I. Catton, Convection in a closed rectangular region: The onset of motion, J. Heat Transfer 92, 186 (1970).

[63] I. Catton, The effect of insulating vertical walls on the onset of motion in a fluid heated from below, Int. J. Heat Mass Transfer 15, 665 (1972).

[64] J. Mizushima and O. Matsuda, Onset of 3D thermal convection in a cubic cavity, J. Phys. Soc. Jpn. 66, 2337 (1997).
[65] A. Mialdun and V. Shevtsova, Digital interferometry as a powerful tool to study the thermodiffusion effect, C. R. Mécanique 339, 362 (2011).

[66] Y. Kanda, E. Shoji, L. Chen, J. Okajima, A. Komiya, and S. Maruyama, Visualization of heat and mass transfer near the formation and dissociation interface of $\mathrm{CO} 2$ hydrate with high-speed phase-shifting interferometer, Int. Heat Transfer Conf. 2018, 8767 (2018).

[67] D. Y. Arifin, L. Y. Lee, and C. H. Wang, Mathematical modeling and simulation of drug release from microspheres: Implications to drug delivery systems, Adv. Drug Deliv. Rev. 58, 1274 (2006).

[68] H. B. Kim, J. Hertzberg, C. Lanning, and R. Shandas, Noninvasive measurement of steady and pulsating velocity profiles and shear rates in arteries using echo PIV: In vitro validation studies, Ann. Biomed. Eng. 32, 1067 (2004).

[69] M. F. Haller and W. M. Saltzman, Localized delivery of proteins in the brain: Can transport be customized? Pharm. Res. 15, 377 (1998).

[70] J. E. Millerd, N. J. Brock, J. B. Hayes, M. B. North-Morris, M. Novak, and J. C. Wyant, Pixelated phase-mask dynamic interferometer, Interferometry XII: Tech. Anal. 5531, 304 (2004).

[71] A. Martin-Mayor, M. M. Bou-Ali, M. Aginagalde, and P. Urteaga, Microfluidic separation processes using the thermodiffusion effect, Int. J. Therm. Sci. 124, 279 (2018).

[72] G. Qiu, Z. Gai, Y. Tao, J. Schmitt, G. A. Kullak-Ublick, and J. Wang, Dual-functional plasmonic photothermal biosensors for highly accurate severe acute respiratory syndrome coronavirus 2 detection, ACS Nano 14, 5268 (2020). 